\section{A) Check for updates}

Cite this: Nanoscale, 2020, 12, 9756

\title{
Actuated plasmonic nanohole arrays for sensing and optical spectroscopy applications $\dagger$
}

\author{
Daria Kotlarek, $\ddagger^{\mathrm{a}}$ Stefan Fossati, ${ }^{a}$ Priyamvada Venugopalan, $\S^{\mathrm{a}, \mathrm{b}}$ \\ Nestor Gisbert Quilis, ${ }^{a}$ Jiři Slabý, ${ }^{c}$ Jiři Homola, (D) ${ }^{c}$ Médéric Lequeux, ${ }^{d}$ \\ Frédéric Amiard, ${ }^{e}$ Marc Lamy de la Chapelle, ${ }^{e}$ Ulrich Jonas ${ }^{f}$ and Jakub Dostálek (D)*a
}

\begin{abstract}
Herein, we report a new approach to rapidly actuate the plasmonic characteristics of thin gold films perforated with nanohole arrays that are coupled with arrays of gold nanoparticles. The near-field interaction between the localized and propagating surface plasmon modes supported by the structure was actively modulated by changing the distance between the nanoholes and nanoparticles and varying the refractive index symmetry of the structure. This approach was applied by using a thin responsive hydrogel cushion, which swelled and collapsed by a temperature stimulus. The detailed experimental study of the changes and interplay of localized and propagating surface plasmons was complemented by numerical simulations. We demonstrate that the interrogation and excitation of the optical resonance to these modes allow the label-free SPR observation of the binding of biomolecules, and is applicable for in situ SERS studies of low molecular weight molecules attached in the gap between the nanoholes and nanoparticles.
\end{abstract}

Received 28th January 2020,

Accepted 30th March 2020

DOI: $10.1039 / \mathrm{d} 0 \mathrm{nr} 00761 \mathrm{~g}$

rsc.li/nanoscale organelles. ${ }^{21}$ Moreover, NHA have enabled studies on lipid membranes that span over the holes ${ }^{22}$ and allowed the facile incorporation of membrane proteins for interaction studies with drug candidates. ${ }^{16,23}$

NHA structures enable the electromagnetic field to be strongly confined inside subwavelength nanoholes ${ }^{24}$ due to the excitation of two types of surface plasmon modes, propagating surface plasmons (PSPs, also referred to as surface plasmon polaritons) traveling along the metal surface and localized surface plasmons (LSPs), which occur at the sharp edges of the holes. The coupling to these modes can be tailored for specific purposes by controlling the structure geometry, including hole shape, diameter, and lateral periodic or quasi-periodic spacing. ${ }^{25}$ In addition, a more complex spectrum of supported plasmonic modes can be utilized by combining complementary geometries that support LSP modes at similar wavelengths based on Babinet's principle. ${ }^{26}$ When the NHA geometry approaches that of complementary metallic nanoparticle (NP) arrays, additional LSP resonance is introduced, which can near-field couple with the NHA. ${ }^{27}$ Moreover, NHA structures that comprise stacks of periodically perforated metallic films ${ }^{28}$ and NHA + NP structure with a defined lateral offset of NPs with respect to the nanohole center ${ }^{29}$ have been investigated. Herein, we report a new approach to rapidly actuate the plasmonic characteristics of thin gold films perforated with nanohole arrays by thermo-responsive hydrogel and demonstrate its utilization to sensing with flow-through format SPR and SERS readout. ${ }^{30}$ It is worth noting that arran- 
a)

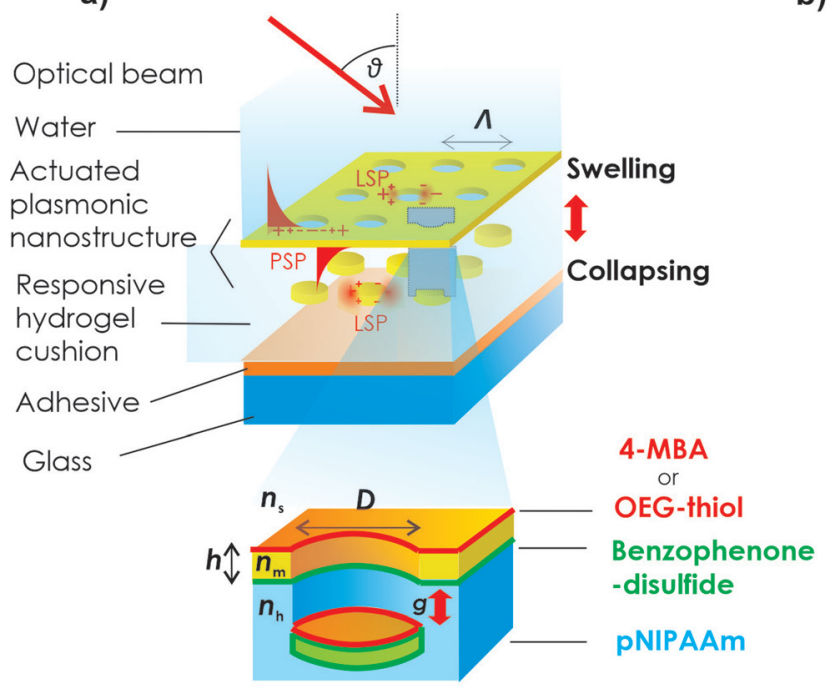

b)

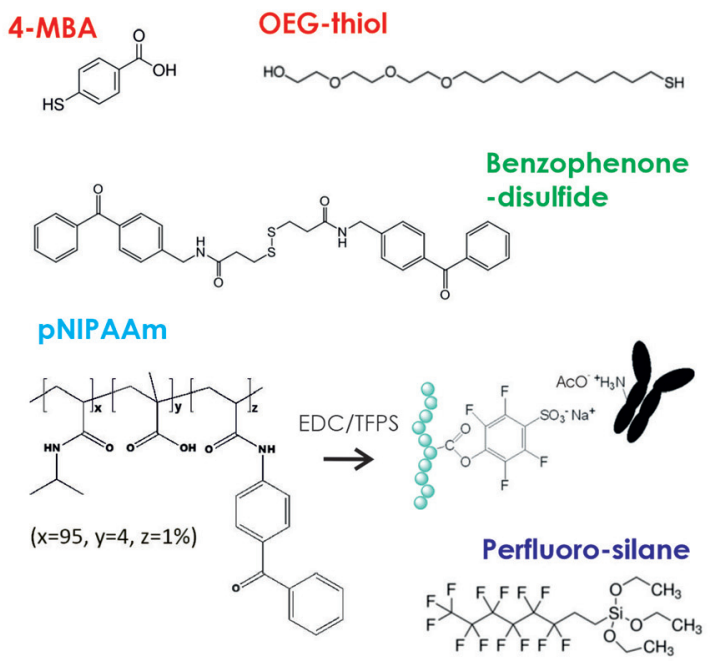

Fig. 1 (a) Schematics of plasmonic nanostructure composed of periodic nanohole arrays (NHA) coupled with arrays of nanoparticles (NP) by the use of responsive hydrogel cushion implemented by materials with the chemical structure shown in (b).

ging the metallic nanoholes and nanoparticles in periodic arrays also decreases the damping of plasmonic modes by their diffraction coupling through the formation of the socalled surface lattice resonances. ${ }^{31}$

Perforated metal layer structures with NHA have been recently prepared using a range of lithography processes. In top-down approaches, a variety of geometries become accessible using focused ion beam milling (FIB) and electron beam lithography (EBL). ${ }^{32,33}$ Nanoimprint lithography and template stripping $^{34}$ are used to partially alleviate the limited throughput of EBL and FIB by the replication of the NHA structure from pre-fabricated templates. Alternatively, NHAs were also prepared using bottom-up approaches, mostly relying on the self-organization of colloid particles. ${ }^{35}$ Using sub-monolayer surface coverage of colloids, the colloid particles are used as a mask for the preparation of sparse and disordered nanoholes, while dense monolayers of colloids are employed to create well-ordered nanohole geometries. ${ }^{28,29,36}$ This approach allows the pattern period, $\Lambda$, to be controlled by the size of the colloid particles and the hole diameter, $D$, can be independently adjusted via isotropic etching of the assembled particles before metal deposition. This technique was further extended for the preparation of nanoholes on cavities, ${ }^{37}$ and a lift-off approach of the NHA membrane was also adopted for its subsequent transfer to more complex structures with multiple stacked patterned metallic layers. $^{28}$

Typically, NHAs used for sensing applications are prepared via stripping-based techniques or lithographic methods directly on a non-permeable solid support, which is loaded to a microfluidic device. ${ }^{38,39}$ However, the liquid sample to be analyzed is flowed over the perforated NHA surface with closed-ended pores, where the liquid flow velocity is equal to zero, and consequently, the transport of molecules to and inside the pores is dominantly driven by slow diffusion. ${ }^{40,41}$
Thus, to overcome this limitation, there a flow-through assay format has been developed, in which the analyte solution is transported internally across the nanoporous film. ${ }^{8,16,41,42}$ However, these experiments typically rely on the structure of the NHA prepared on thin nitride membranes, which requires multiple lithography steps and complicates their application in sensing experiments.

The present study demonstrates a new type of NHA + NP structure, which is supported by a thermo-responsive hydrogel cushion. It is made from an $N$-isopropylacrylamide hydrogel material that can expand and contract in an aqueous environment, thereby actuating the plasmonic properties of metallic nanostructures. ${ }^{43-45}$ In this architecture, the hydrogel cushion accommodates arrays of gold NPs, which are located below the NHA and their mutual distance, $g$, can be on demand actuated (Fig. 1). Herein, we investigate the spectra of supported PSP and LSP modes and their spectral detuning by temperatureinduced reversible swelling and collapsing of a hydrogel cushion. In addition, the hydrogel can serve as a three-dimensional binding matrix for the immobilization of bio-functional molecules and the applicability of the structure for SPR (surface plasmon resonance) and SERS (surface-enhanced Raman scattering) detection is demonstrated with the use of plasmonic modes that probe the open pores, through which aqueous samples can be actively flowed.

\section{Results and discussion}

We developed a method to prepare a nanostructure geometry that combines thin gold films perforated with NHA and arrays of gold NP, which are suspended in a thin layer of a $\operatorname{poly}(\mathrm{N}$-isopropyl acrylamide) (pNIPAAm)-based hydrogel. It serves as a responsive cushion, which responds to temperature changes 
since pNIPAAm exhibits a lower critical solution temperature (LCST) of $32^{\circ} \mathrm{C}$. Below its LCST, it is hydrophilic, and it contains large amounts of water in its polymer network structure. When the temperature increases above its LCST, it abruptly collapses by expelling water. In the nanostructure geometry (Fig. 1a), the pNIPAAm-based hydrogel cushion was allowed to swell and collapse via the diffusion of water through the NHA pores and its volume changes were utilized for active control of the distance, $g$, between NHA and NP. In addition, the hydrogel was made from a terpolymer that carries pendant groups attached to its backbone, enabling its post-modification with bio-functional molecules (Fig. 1b) for application in optical spectroscopy and biosensors. ${ }^{46}$ It is worth noting that this thermo-responsive hydrogel cushion allowed the controlled opening and closing of the nanohole arrays of pores, and switching to the open state was accompanied by the rapid diffusion of water, which drags contained biomolecules through the pores, where a plasmonic hotspot occurs. The spectrum of plasmonic modes probing the pores of the structure and their near field coupling was investigated in detail, as follows.

\section{Preparation of NHA + NP structure with hydrogel cushion}

The NHA + NP structure featuring actively tunable plasmonic properties was prepared by a combination of UV nanoimprint lithography (UV-NIL) and template-stripping (Fig. 2a). Arrays of nanopillars cast to the transparent OrmoStamp material were used as a template. AFM observation of the structure topography showed that the arrays of nanopillars exhibited a diameter of $D=100 \mathrm{~nm}$, height of $100 \mathrm{~nm}$, and they were arranged in rectangular arrays with a set period of $\Lambda=460 \mathrm{~nm}$ (Fig. 2b). The arrays of OrmoStamp nanopillars were then activated by UV-ozone treatment and modified with perfluoro-silane using vapor deposition to reduce their surface energy. Subsequently, the nanopillars with a perfluoro-silane anti-adhesive layer were coated by a gold layer with a thickness of $h=50 \mathrm{~nm}$. SEM observation (Fig. 2c) revealed that the gold deposition led to the formation of a continuous layer, which is protruded by the nanopillars and their top is capped by gold that is not connected to the bottom continuous gold layer. Afterward, the outer gold surface was modified by a self-assembled monolayer of photo-active benzophenone-disulfide, and subsequently coated with a pNIPAAm-based terpolymer layer. This terpolymer contains the same photo-reactive benzophenone groups in its backbone (see Fig. 1b) and upon irradiation with UV light these chains were simultaneously covalently crosslinked and attached to the gold via the benzophenone-disulfide linker. Then, the outer surface of the crosslinked pNIPAAmbased polymer was pressed against a glass substrate with a soft adhesive layer (Ostemer resin pre-cured with UV light), which was subsequently thermally cured overnight at a temperature of $50{ }^{\circ} \mathrm{C}$. Finally, the assembly was stripped at the OrmoStamp-gold interface (treated with anti-adhesive layer) to yield a structure with a thin gold film perforated by NHA, which were attached to the pNIPAAm-based crosslinked polymer networks and underneath comprised of embedded gold NPs spatially separated from the perforated continuous gold film. The AFM topography image in Fig. 2d shows that the pores exhibit the same diameter, $D$, as the nanopillars and the SEM image of an edge of the structure in Fig. 2e confirms that under the NHA, arrays of gold NPs are present (which were stripped from the top of the OrmoStamp pillars). It is worth noting that the distortions of the surface that are visible in Fig. 2e are a result of breaking the sample to obtain the cross-section image.

\section{Optical observation of plasmonic modes}

The spectra of the LSP and PSP modes supported by the prepared nanostructure were investigated via optical transmission measurements. To distinguish between the diffraction coupling to the dispersive PSP modes (traveling along the gold film) and non-dispersive LSP resonances (supported by the pores in the NHA + NP nanostructure), transmission spectra were measured via collimated beam impinging on the NHA $+\mathrm{NP}$ structure at angles in the range of $\theta=0^{\circ}$ to $25^{\circ}$. The transmitted beam emitted from a halogen light bulb was then analyzed with a spectrometer in the wavelength range of $\lambda=$ 500-850 nm. Firstly, the wavelength-angular dependence of the transmission was measured for a structure that was dry and in contact with air (with a refractive index of $n_{\mathrm{s}}=1$ ). As presented in Fig. 3a, the acquired spectrum shows that the excitation of non-dispersive resonance is manifested as a dip centered at a wavelength of $\lambda_{\mathrm{A}}=600 \mathrm{~nm}$. Moreover, an additional dispersive mode occurs, and its excitation is associated with the dip in the transmission spectrum at $\lambda_{\mathrm{C}}^{\mathrm{d}}=$ $750 \mathrm{~nm}$, which splits when the angle of incidence, $\theta$, deviates from zero. Secondly, the structure was clamped to a flow-cell and water (with a refractive index of $n_{\mathrm{s}}=1.33$ ) was flowed over its surface, which was kept at a temperature of $T=40^{\circ} \mathrm{C}$. This temperature is above the LCST of pNIPAAm, and thus this material exhibits hydrophobic properties, preventing the diffusion of water into the polymer networks through the NHA pores. Since the refractive index of the dielectric above the structure $n_{\mathrm{s}}$ increased, a new dispersive dip resonance centered at a wavelength of $\lambda_{\mathrm{B}}^{\mathrm{d}}=650 \mathrm{~nm}$ appeared, while the resonance features $\lambda_{\mathrm{A}}$ and $\lambda_{\mathrm{C}}^{\mathrm{d}}$ changed negligibly (Fig. 3b). Thirdly, the structure in contact with water was cooled to $T=22{ }^{\circ} \mathrm{C}$, which is below the LCST of pNIPAAm. Then, a strong blueshift in the $\lambda_{\mathrm{A}}$ and $\lambda_{\mathrm{C}}^{\mathrm{d}}$ resonances occurred in the transmission spectrum, and also much weaker spectral shift of $\lambda_{\mathrm{B}}^{\mathrm{d}}$ accompanied by a decrease in coupling efficiency at this wavelength (Fig. 3c). These observations indicate that the resonances at $\lambda_{\mathrm{A}}$ and $\lambda_{\mathrm{C}}^{\mathrm{d}}$ are associated with the coupling to the surface plasmon modes that confine the electromagnetic field in the inner side of the structure in contact with the pNIPAAm-based hydrogel (with a refractive index of $n_{\mathrm{h}}=1.47$ at $T=22{ }^{\circ} \mathrm{C}$ and $n_{\mathrm{h}}=1.37$ at $T=40{ }^{\circ} \mathrm{C}$, see ESI, Fig. S1 $\dagger$ ). The resonance $\lambda_{\mathrm{B}}^{\mathrm{d}}$ probes the upper part of the structure in contact with water $\left(n_{\mathrm{s}}\right.$ $=1.33$ ), which changes its refractive index with temperature much less than pNIPAAm. Since the resonances at $\lambda_{B}^{d}$ and $\lambda_{C}^{d}$ are dispersive, they can be attributed to the PSP modes travelling at the upper or bottom interface of the gold film, respect- 
a)

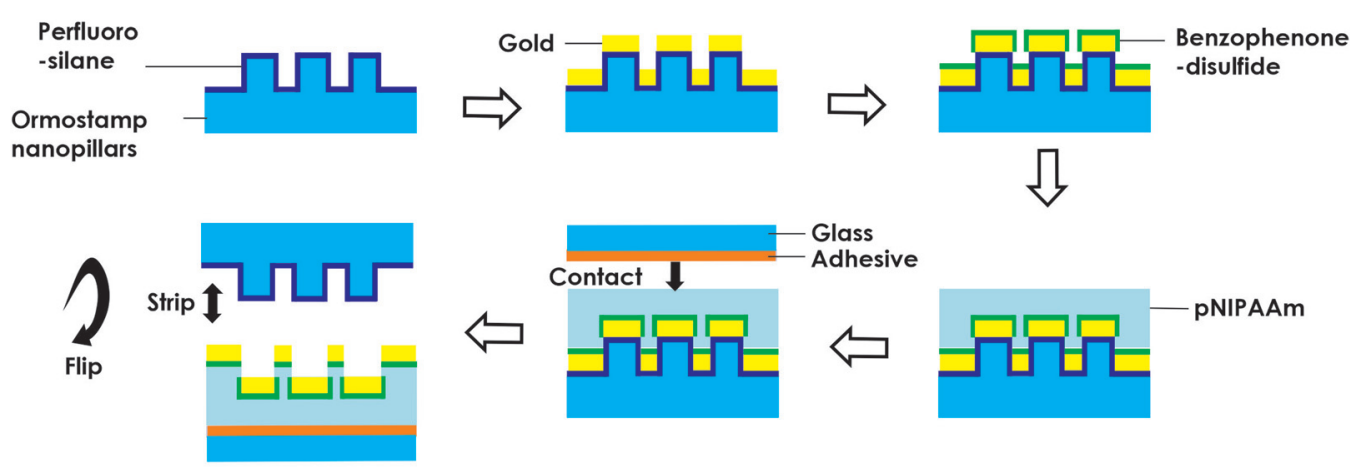

b)

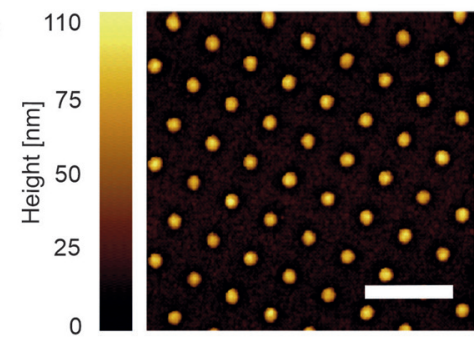

d)

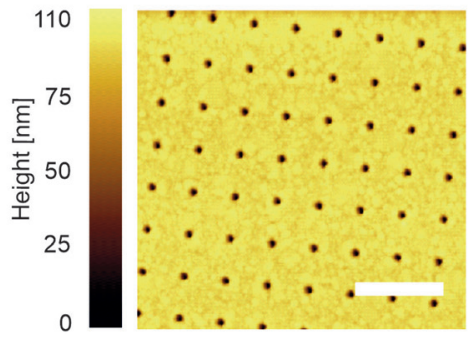

c)

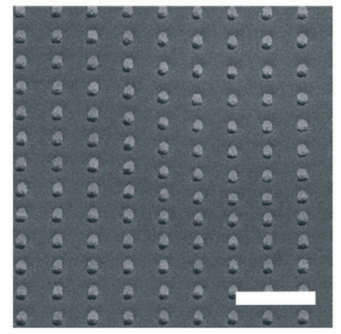

e)

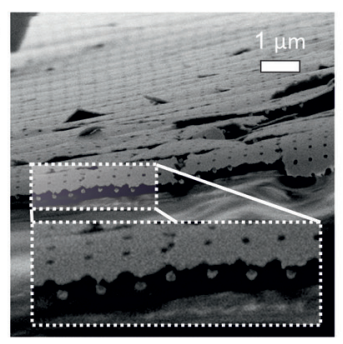

Fig. 2 (a) Schematic of the preparation steps of the investigated NHA + NP nanostructure, (b) AFM image of the template with arrays of nanopillars cast to OrmoStamp, (c) SEM image after coating of the template with a $50 \mathrm{~nm}$ thick gold layer, (d) AFM image of the topography of the stripped surface with gold NHA, and (e) SEM image of a broken edge of the NHA + NP structure tethered to a solid surface via the responsive pNIPAAmbased polymer. All scale bars are $1 \mu \mathrm{m}$ in length.

ively. The non-dispersive nature of the $\lambda_{\mathrm{A}}$ resonance indicates it can be ascribed to the LSP mode.

A more detailed dependence of the transmission spectra on temperature $T$ was investigated for the normal angle of incidence $\theta=0$ (Fig. 3d). The spectra were measured using a different light source (supercontinuum laser) in order to extend the wavelength range to $900 \mathrm{~nm}$, which allowed us to observe an additional feature manifested as a transmission peak at $\lambda_{\mathrm{C}}^{\mathrm{p}}=810 \mathrm{~nm}$. Moreover, this showed more clearly that close to $\lambda_{\mathrm{B}}^{\mathrm{d}}$, a peak appeared at a wavelength $\lambda_{\mathrm{B}}^{\mathrm{p}}$. It is worth noting that the measured transmission spectra were normalized with that obtained for a flat $50 \mathrm{~nm}$ thick gold film, which exhibited a rapidly decreasing transmission with wavelength in the red and near infrared region of the spectrum. Therefore, the measurement of absolute transmission values was not possible, and thus only relative values are presented. In addition, the spectral positions of the resonances in Fig. 3a, b and d slightly differ since they were measured with different (although fabricated under identical conditions) NHA + NP nanostructures.

\section{Identification of plasmonic modes aided by simulations}

The five observed resonant features in the measured transmission spectra were identified using numerical finite difference time domain (FDTD) simulations. This model was employed to calculate the absorption wavelength spectra and near-field distribution of the electromagnetic field occurring in the structure upon a plane wave normally impinging at its surface. The simulations of the absorption spectra allowed us to distinguish the resonant excitation of the LSP and PSP modes (which is accompanied with damping) from other features occurring in the transmission spectrum, which are related to the interference between multiple specular and nonspecular diffracted beams and their falling after the horizon in the superstrate or substrate. As shown in Fig. 4a (blue curve), we initially analyzed a structure composed of only arrays of 
a)

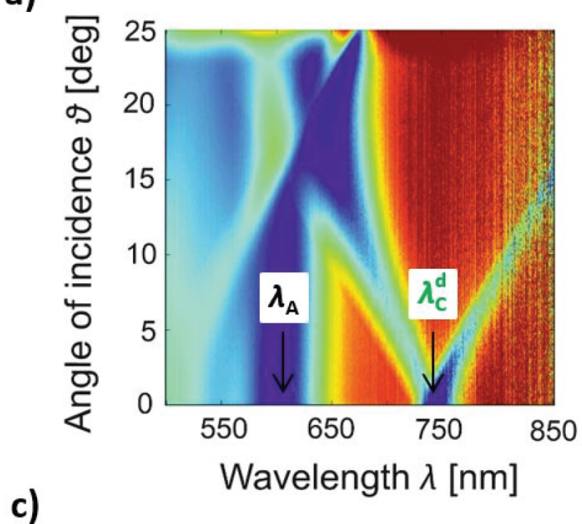

c)

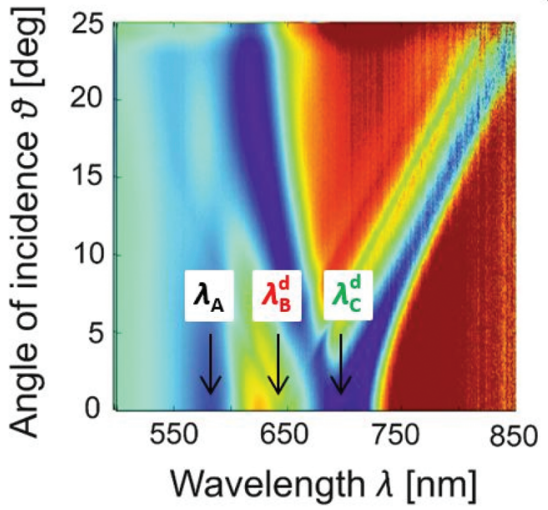

b)

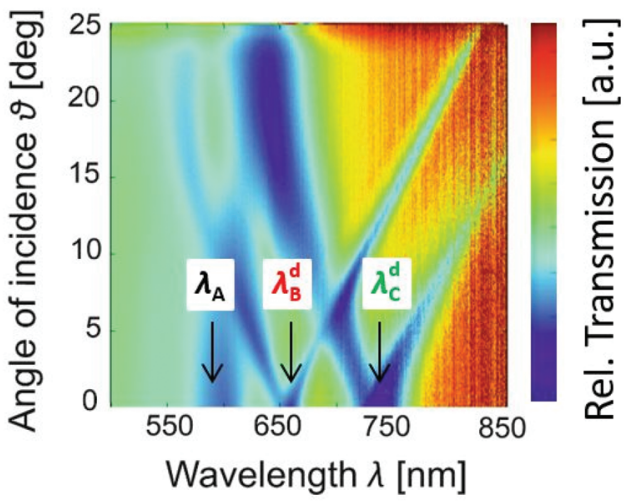

d)

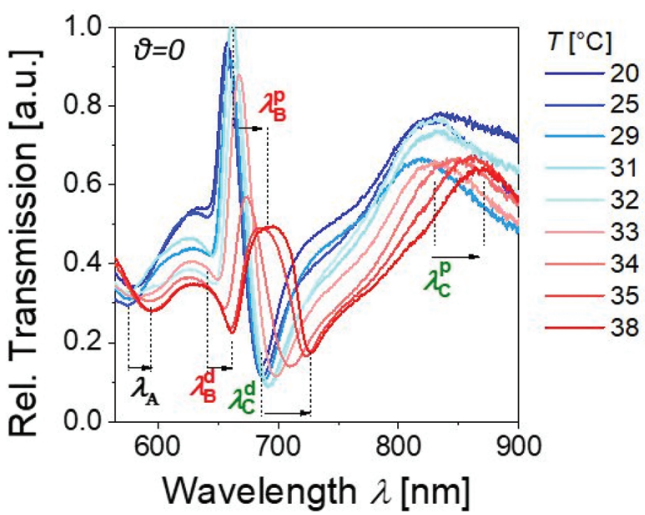

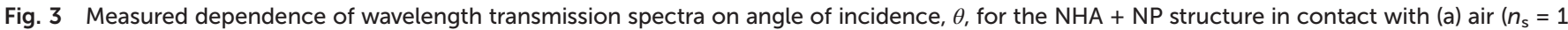

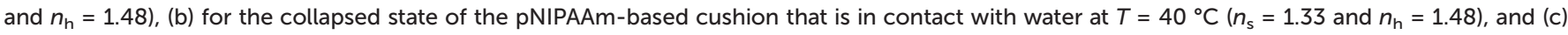

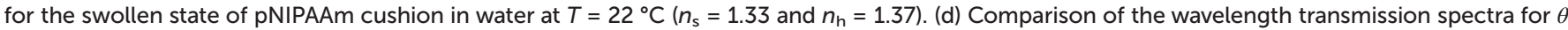

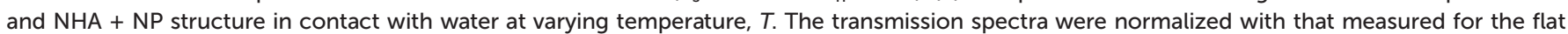
structure without the perforated Au film.

cylindrically shaped nanoparticles (NPs with a height of $h=$ $50 \mathrm{~nm}$, average diameter of $D=100-120 \mathrm{~nm}$, and period of $\Lambda=$ $460 \mathrm{~nm}$ ) embedded between dielectrics with the refractive indices of $n_{\mathrm{h}}=1.47$ and $n_{\mathrm{s}}=1.33$. The simulated spectrum reveals that the resonance associated with the coupling to LSP on the arrays of NPs is manifested as a strong absorption peak at a wavelength of about $707 \mathrm{~nm}$. The complementary NHA structure was composed of a $50 \mathrm{~nm}$ thick gold film that was perforated with arrays of cylindrical nanoholes exhibiting the same diameter $D$ and sandwiched between the same dielectrics with refractive indices of $n_{\mathrm{h}}=1.47$ and $n_{\mathrm{s}}=1.33$. The absorption spectrum in Fig. 4a (red curve) shows three resonances located at the wavelengths of $\lambda_{\mathrm{A}}=630 \mathrm{~nm}, \lambda_{\mathrm{B}}=676 \mathrm{~nm}$, and $\lambda_{\mathrm{C}}=774 \mathrm{~nm}$. At these wavelengths, the near-field distribution of the electric field amplitude $|E|$ (normalized with that of the incidence plane wave $\left|E_{0}\right|$ ) was simulated, as can be observed in the right part of Fig. 4a. These plots reveal that the resonance at wavelength $\lambda_{\mathrm{A}}$ exhibits the characteristics of the dipolar LSP mode, which confines the field inside the nanohole. The resonance at the longer wavelength $\lambda_{\mathrm{B}}$ shows a more delocalized field profile on the top interface of the gold film with the superstrate $n_{\mathrm{s}}=1.33$, which confirms it is due to firstorder diffraction coupling to the traveling PSP mode at this surface. The resonance at the NIR wavelength $\lambda_{\mathrm{C}}$ is accompanied with the confinement of the electric field at the bottom gold layer interface with the dielectric $n_{\mathrm{h}}=1.47$, which peaks at the mouth of the pore and its distribution suggests the main origin corresponds to the first-order diffraction coupling to the PSPs traveling along the bottom gold film surface. It should be noted that these simulations were carried out for the mouth of the nanopores filled by a dielectric with a refractive index $n_{\mathrm{s}}$ since the fabrication procedure involving stripping from arrays of nanopillars suggests this geometry (see Fig. 2a).

The simulations in Fig. 4b (brown curve) reveal that the short wavelength resonance was blue-shifted to $\lambda_{\mathrm{A}}=621 \mathrm{~nm}$, the middle resonance blue-shifted to $\lambda_{\mathrm{A}}=672 \mathrm{~nm}$, and the long-wavelength resonance red-shifted to $\lambda_{\mathrm{C}}=813 \mathrm{~nm}$ after the coupling of the nanohole arrays with the cylindrical nanoparticle arrays (NHA + NP). In these simulations, the gap distance between the bottom edge of the nanopore and the upper surface of the cylindrically shaped nanoparticle was set to $g=$ $50 \mathrm{~nm}$, which corresponds to the difference between the nanopillar height of $100 \mathrm{~nm}$ and the thickness of the gold layer of $h=50 \mathrm{~nm}$ (see Fig. 1 and 2). When the refractive index of the superstrate decreased from $n_{\mathrm{s}}=1.33$ (representing 
a)

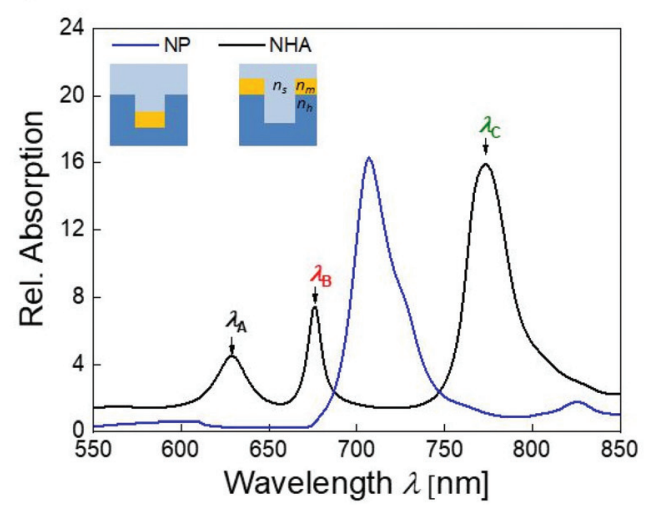

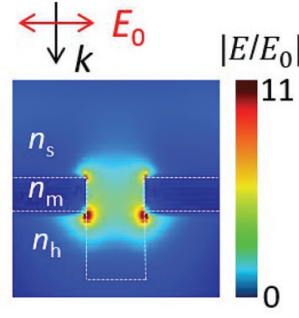

$\lambda_{\mathrm{A}}$
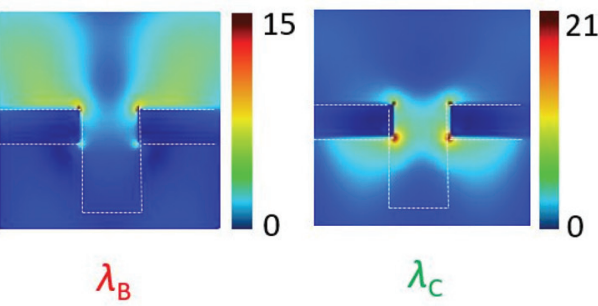

b)
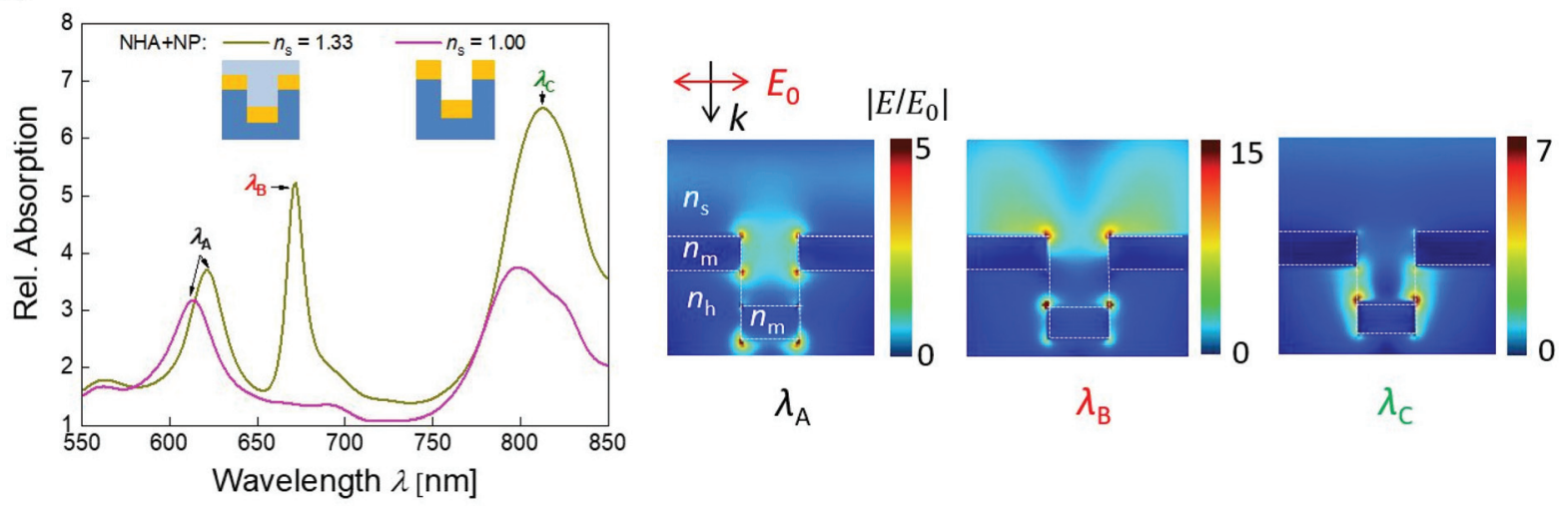

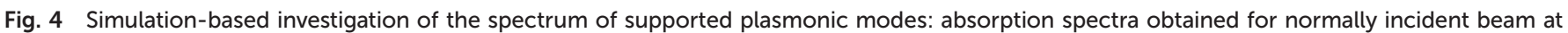

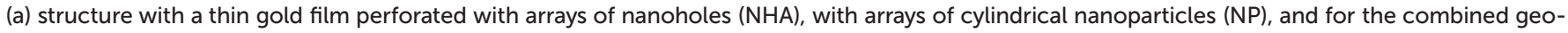

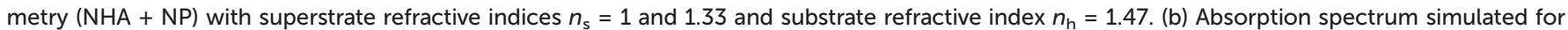

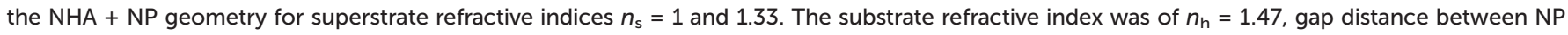

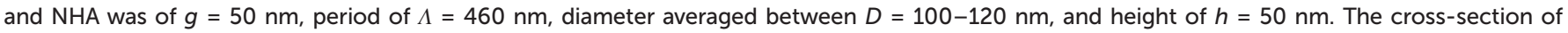
spatial distribution of the electric field amplitude was simulated for the plasmonic modes as indicated in the inset.

water) to $n_{\mathrm{s}}=1$ (representing air), the middle-wavelength resonance disappeared, as shown in Fig. $4 \mathrm{~b}$ (green curve). This observation agrees with the measurements presented in Fig. 3 and confirms that the middle resonance occurs due to the first-order diffractive coupling to the PSP mode at the outer gold layer surface, which is the most sensitive to variations in refractive index on the upper interface (superstrate $n_{\mathrm{s}}$ ). In addition, this refractive index decrease led to a slight blue shift in $\lambda_{\mathrm{A}}$ and $\lambda_{\mathrm{C}}$ since the field distribution corresponding to these resonances also partially probe the dielectric $n_{\mathrm{s}}$. The spatial profile of the near field-enhanced electric field amplitude in the right part of Fig. 4b shows that the presence of gold disk nanoparticles slightly perturbed the nanohole LSP resonance at $\lambda_{\mathrm{A}}$ as the field is dragged to the bottom part of the disk nanoparticle. The resonance at $\lambda_{\mathrm{B}}$ due to the PSPs traveling on the top interface only weakly couples with the disk nanoparticles, contrary to the bottom PSP mode $\lambda_{\mathrm{C}}$, which exhibits a field distribution with a more pronounced confinement in the gap.

Interestingly, the simulations predicted that only three plasmonic modes are supported in the investigated wavelength range (Fig. 4b, brown curve) when the geometry of NHA (with three plasmonic modes, black curve Fig. 4a) and NP arrays (with one mode, blue curve in Fig. 4a) are combined to yield the experimentally investigated structure NHA + NP. However, five features were identified in the experimental transmission data presented Fig. 3, which is greater than the number of predicted plasmonic modes. The discrepancy between the simulated absorption spectra and experimentally measured specular transmission can be explained by the Fano shape of the two measured transmission resonances. The middle wavelength peak at $\lambda_{\mathrm{B}}^{\mathrm{p}}$ and $\operatorname{dip}$ at $\lambda_{\mathrm{B}}^{\mathrm{d}}$ can be attributed to the excitation of a single PSP mode at the interface of the structure with superstrate $n_{\mathrm{s}}$ and its asymmetric shape can be ascribed to the interference with additional waves generated by the structure in a broader wavelength range (previously observed for related plasmonic nanostructure by simulations ${ }^{3}$ ). Similarly, the long wavelength peak at $\lambda_{\mathrm{C}}^{\mathrm{p}}$ and $\operatorname{dip}$ at $\lambda_{\mathrm{C}}^{\mathrm{d}}$ can be attributed to the excitation of the PSP mode at the opposite interface of the structure with superstrate $n_{\mathrm{h}}$.

In the next step, we performed more detailed simulations to reveal the spectral detuning of the resonances by the refrac- 

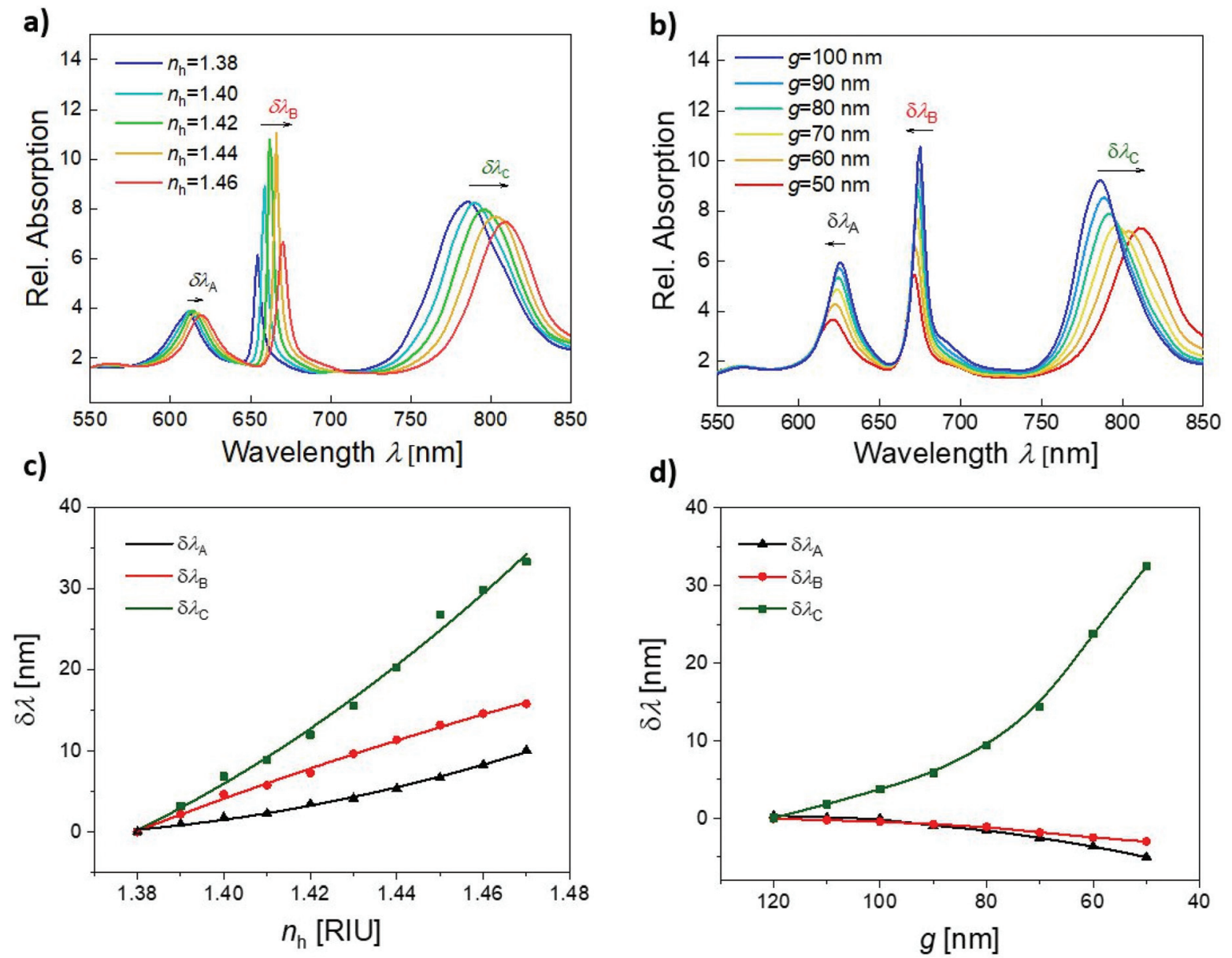

Fig. 5 Simulated absorption spectra for the structure NHA + NP for varying: (a) refractive index of substrate $n_{\mathrm{h}}$ and (b) distance $g$ between NHA and NP. From these spectra, the spectral shift of three resonant features were determined for changes in (c) $n_{\mathrm{h}}$ and (d) $g$. The superstrate refractive index was set to $n_{\mathrm{s}}=1.33$, the substrate refractive index for (b) and (d) was $n_{\mathrm{h}}=1.47$, gap distance between NP and NHA for (a) and (c) was set as $g=$ $50 \mathrm{~nm}$, period was $\Lambda=460 \mathrm{~nm}$, average diameter between $D=100-120 \mathrm{~nm}$, and height $h=50 \mathrm{~nm}$.

tive index changes of the bottom dielectric $n_{\mathrm{h}}$ and distance $g$ between the gold NPs and NHA. These simulations represent the expected effect of the swelling and collapsing of the pNIPAAm-based hydrogel cushion. In general, an increase in swelling is assumed to be accompanied with a prolongation of distance $g$, a decrease in polymer volume content, and consequently a decrease in the refractive index $n_{\mathrm{h}}$. Fig. $5 \mathrm{a}$ and $\mathrm{b}$ show that the refractive index $n_{\mathrm{h}}$ gradually increased from 1.38 to 1.46 and distance $g$ varied between 50 and $100 \mathrm{~nm}$, respectively. The increase in the substrate refractive index $n_{\mathrm{h}}$ led to a redshift for all three resonances (Fig. 5a), which is consistent with the experimental data presented in Fig. 3d. For small changes in the refractive index, the variations in the resonant wavelengths can be assumed to be linear, and accordingly, the determined refractive index sensitivity of $\delta \lambda_{\mathrm{B}} / \delta n_{\mathrm{h}}=190 \mathrm{~nm}$ $\mathrm{RIU}^{-1}$ and $\delta \lambda_{\mathrm{C}} / \delta n_{\mathrm{h}}=390 \mathrm{~nm} \mathrm{RIU}^{-1}$ were obtained from the simulated data. These values are in the range reported for another SPR sensor configuration, which utilizes grating coupling to PSP modes. ${ }^{47}$ The lower sensitivity of $\delta \lambda_{\mathrm{A}} / \delta n_{\mathrm{h}}=90 \mathrm{~nm}$ $\mathrm{RIU}^{-1}$ for the LSP mode is also in accordance with the previous observations on LSPR. ${ }^{44}$ Similarly, the refractive index sensitivity of the three plasmon modes to a change in the refractive index of the superstrate $n_{\mathrm{s}}$ was determined to be $\delta \lambda_{\mathrm{A}} / \delta n_{\mathrm{S}}=$ $137 \mathrm{~nm} \mathrm{RIU}{ }^{-1}, \delta \lambda_{\mathrm{B}} / \delta n_{\mathrm{S}}=260 \mathrm{~nm} \mathrm{RIU}^{-1}$ and $\delta \lambda_{\mathrm{C}} / \delta n_{\mathrm{S}}=83 \mathrm{~nm}$ $\mathrm{RIU}^{-1}$ according to the data presented in Fig. S2.† Apparently, the highest sensitivity is observed for the mode at $\lambda_{\mathrm{B}}$, which is ascribed to the PSP at the outer interface, where the field is dominantly confined.

The simulated spectra for varying gap distances $g$ are presented in Fig. 5b. They show more complex behavior and the resonances $\lambda_{\mathrm{A}}$ and $\lambda_{\mathrm{B}}$ are weakly blue-shifted with a decrease in distance $g$, while $\lambda_{\mathrm{C}}$ is strongly red-shifted. These changes exhibit non-linear behavior, and for the shorter distances of $g$, they are more pronounced than for the longer distances of $g$. Therefore, this observation can be attributed to the near-field coupling between the gold NPs and NHA, which is particularly pronounced for the resonance $\lambda_{\mathrm{C}}$ with its field tightly confined in the gap (see right part of Fig. 4b). Interestingly, for the long distance $g$, an increase in the absorption close to the wavelength of $700 \mathrm{~nm}$ occurred, which may be due to the re-occur- 
rence of the LSP resonance supported by gold NP arrays not coupled with NHA, as presented in Fig. 4 a.

\section{Actuating of plasmonic modes}

The swelling and collapsing of the pNIPAAm-based hydrogel cushion by varying the temperature around its LCST were further exploited for actuating the plasmonic modes supported by the prepared structure NHA + NP. In this experiment, we varied temperature in the range of $T=20-38{ }^{\circ} \mathrm{C}$ and tracked the resonant positions of all the identified spectral features, including the transmission dip at $\lambda_{\mathrm{A}}=577 \mathrm{~nm}$ due to the LSP in the nanoholes, spectral dip at $\lambda_{\mathrm{B}}^{\mathrm{d}}=641 \mathrm{~nm}$ and peak at $\lambda_{\mathrm{B}}^{\mathrm{p}}=$ $658 \mathrm{~nm}$, which are ascribed to the Fano resonance of the PSPS on the top NHA surface, and spectral dip at $\lambda_{\mathrm{C}}^{\mathrm{d}}=684 \mathrm{~nm}$ and peak at a wavelength of $\lambda_{\mathrm{C}}^{\mathrm{p}}=825 \mathrm{~nm}$ attributed to the Fano resonance of PSP at the bottom NHA surface coupled with arrays of NPs (measured at temperature $T=20{ }^{\circ} \mathrm{C}$ ). The obtained response was measured with time upon a step-wise increase and decrease in temperature $T$ according to the analysis of the spectra presented in Fig. 3d. The obtained time kinetics in Fig. 6 show that the changes are reversible. The features presented in Fig. 6a show a gradual increase in spectral position with an increase in temperature $T$. The Fano resonance features $\lambda_{\mathrm{B}}^{\mathrm{d}}$ and $\lambda_{\mathrm{B}}^{\mathrm{p}}$ exhibit similar shifts and the maximum change of about $20 \mathrm{~nm}$ occurred when the temperature increased from $T=22{ }^{\circ} \mathrm{C}$ to $38^{\circ} \mathrm{C}$. The highest slope in the shift occurred close to the LCST of pNIPAAm of $32^{\circ} \mathrm{C}$. At a higher temperature, it was not possible to track the spectral shift in the peak due to the fact that it became weakly pronounced. The spectral dip $\lambda_{\mathrm{C}}^{\mathrm{d}}$ showed the same trend and exhibited a stronger maximum shift of $40 \mathrm{~nm}$ for the temperature increase from $T=22{ }^{\circ} \mathrm{C}$ to $38^{\circ} \mathrm{C}$.
Interestingly, the dependence of $\lambda_{\mathrm{A}}$ and $\lambda_{\mathrm{C}}^{\mathrm{p}}$ shows different behavior. When the temperature increased above $T=22^{\circ} \mathrm{C}$, an initial decrease in the resonance $\lambda_{\mathrm{A}}$ occurred followed by an increase with a local maximum at $T=29^{\circ} \mathrm{C}$, then it decreased again, and above the LCST of pNIPAAm, it rapidly increased and shifted by about $20 \mathrm{~nm}$. The peak position $\lambda_{\mathrm{C}}^{\mathrm{p}}$ showed a complementary trend and it strongly decreased by $15 \mathrm{~nm}$ when the temperature increased from $22{ }^{\circ} \mathrm{C}$ to $27^{\circ} \mathrm{C}$, and then it increased with the local maximum at $30{ }^{\circ} \mathrm{C}$, and when passing the LCST it rapidly redshifted by $38 \mathrm{~nm}$. These anomalous dependencies can be explained by the competing effect of the near-field coupling (which is dominantly controlled by distance $g$ and exhibit non-linear dependence, as shown in Fig. 5d) and refractive index change, which shifts the resonance linearly (see Fig. 5c). The anomalous changes occurred below the LCST of pNIPAAm, which indicates that distance $g$ is not directly proportional to the swelling degree of the hydrogel cushion layer, and other effects such as filling the pores with the swelling polymer networks can occur.

\section{Local probing of molecular binding events}

To explore the potential of the developed hybrid nanostructure for applications in sensing, we employed the plasmonic resonances for local probing of molecular binding events at specific parts. In the first experiment, we directly monitored the binding of biomolecules in the pNIPAAm-based hydrogel cushion from the associated refractive index changes. These changes detuned the SPR wavelengths, where the excitation of the modes probing different the sub-parts of the structure occurred. After the stripping of the structure, the outer gold surface and the mouth of the pores were passivated by the thiol SAM with oligoethylene glycol (OEG) groups. Then, the pNIPAAm-based hydrogel cushion was post-modified in situ by covalent coupling of mouse immunoglobulin G, mIgG. The

\section{a)}

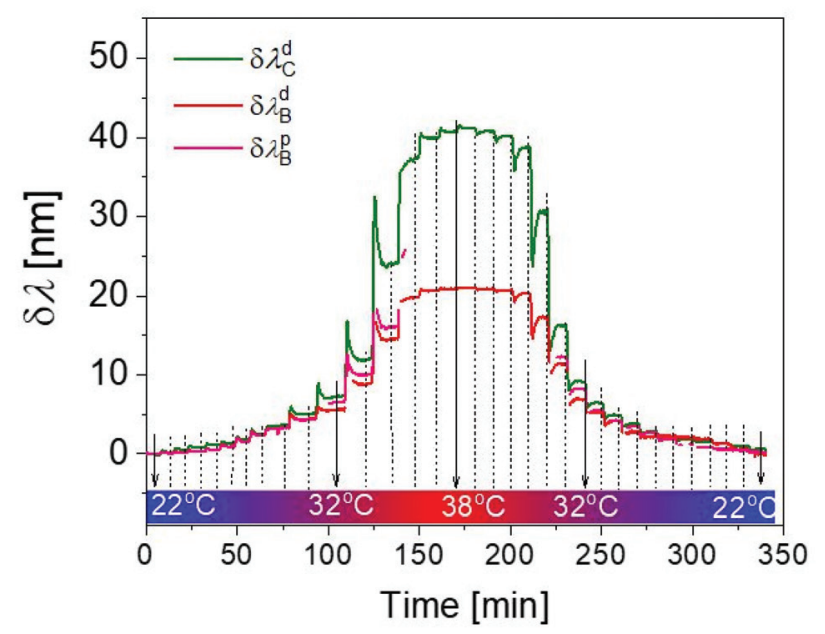

b)

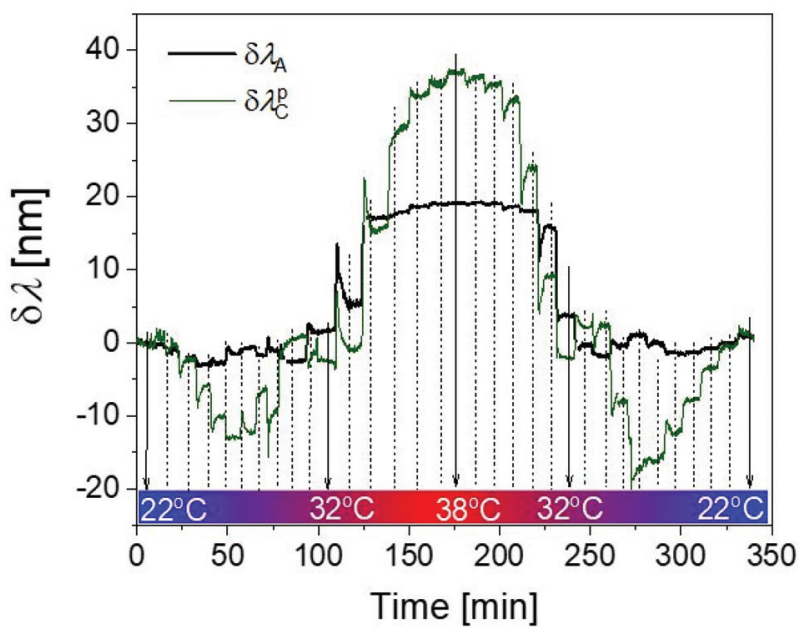

Fig. 6 Dependence of spectral position of plasmonic features plotted as function of time for different temperatures in the range of $T=22{ }^{\circ} \mathrm{C}$ to $T=$ $38^{\circ} \mathrm{C}$ : (a) gradual variation in the spectral positions of dips $\lambda_{\mathrm{B}}^{\mathrm{d}}$ and $\lambda_{\mathrm{C}}^{\mathrm{d}}$ and peak at $\lambda_{\mathrm{B}}^{\mathrm{p}}$ and (b) anomalous variations in the spectral positions of dip $\lambda_{\mathrm{A}}$ and peak $\lambda_{\mathrm{C}}^{\mathrm{p}}$. 
spectral positions of the resonances at $\lambda_{\mathrm{A}}$ and $\lambda_{\mathrm{C}}^{\mathrm{d}}$ were monitored by tracking their spectral detuning upon the surface reactions, as can be seen in Fig. 7a. These two resonances were

a)

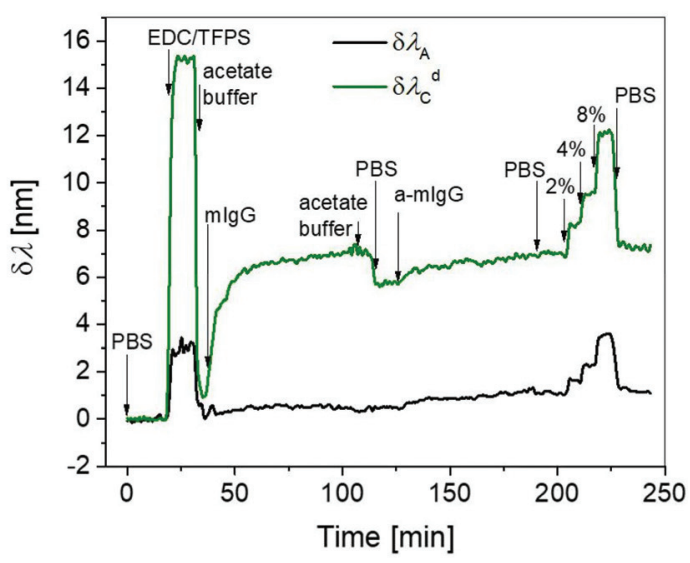

b)

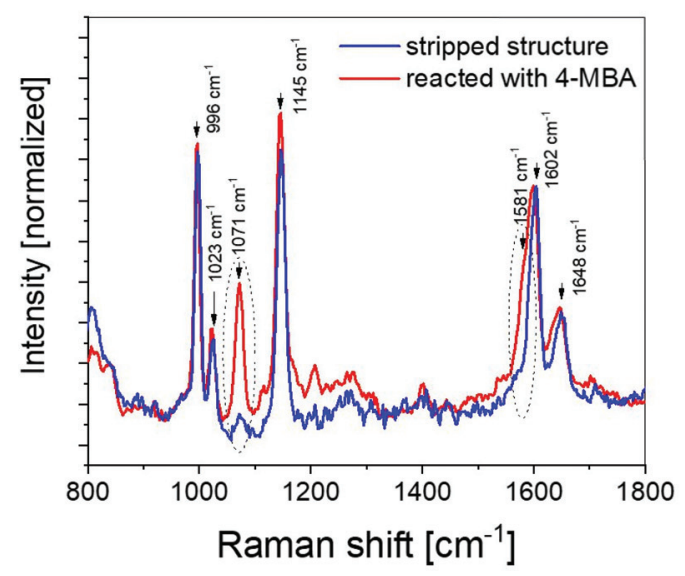

c)

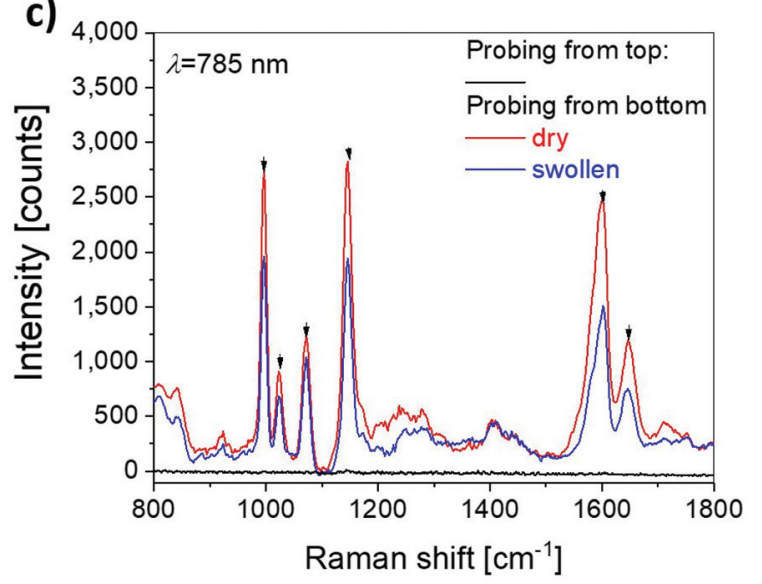

Fig. 7 (a) Probing of covalent coupling of immunoglobulin G molecules (IgG) to pNIPAAm hydrogel polymer networks with the plasmonic modes centred at $\lambda_{A}$ and $\lambda_{C}^{d}$. (b) SERS spectra measured before and after post-modification of the stripped area of gold with 4-MBA, as measured with a laser wavelength of $785 \mathrm{~nm}$. The structure NHA + NP was swollen in water. (c) Comparison of the acquired Raman spectra from the top (dry NHA + NP structure) and bottom (swollen and dry NHA + NP structure). selected since they probe different parts of the structure and are well pronounced in the transmission spectrum. To activate the carboxylic groups within the hydrogel cushion, they were reacted with EDC/TFPS and then a solution with mIgG was flowed over the surface in the time range of 45 to $120 \mathrm{~min}$. After rinsing with a buffer at time $120 \mathrm{~min}$, resonance $\lambda_{\mathrm{C}}^{\mathrm{d}}$ shifted by $6 \mathrm{~nm}$ due to the covalent coupling of mIgG, while resonance $\lambda_{\mathrm{A}}$ showed a much weaker shift of about $0.5 \mathrm{~nm}$. This observation proves that the mIgG molecules could diffuse through the pores and bind to the bottom swollen hydrogel (probed at $\lambda_{\mathrm{C}}^{\mathrm{d}}$ ), while they do not attach to the pores (probed at $\left.\lambda_{\mathrm{A}}\right)$. Then, a series of PBS solutions with sucrose dissolved at a concentration of $2 \%, 4 \%$ and $8 \%$ was flowed over the surface to change the bulk refractive index by $2.8,5.6$ and $11.2 \times 10^{-3}$ RIU, respectively. These low molecular weight molecules did not interact with the structure but freely diffused into the pNIPAAm-based hydrogel, and thus changed the refractive index on both sides of superstrate $n_{\mathrm{s}}$ and substrate hydrogel cushion $n_{\mathrm{h}}$. From the measured shifts in $\lambda_{\mathrm{A}}$ and $\lambda_{\mathrm{C}}^{\mathrm{d}}$, the sensitivity of these resonances was determined to be $\mathrm{d} \lambda_{\mathrm{A}} / \mathrm{d}_{n}=$ $218 \mathrm{~nm} \mathrm{RIU}{ }^{-1}$ and $\mathrm{d} \lambda_{\mathrm{C}}^{\mathrm{d}} / \mathrm{d}_{n} 454 \mathrm{~nm} \mathrm{RIU}{ }^{-1}$, respectively. These values are close to the predicted sum of the sensitivities $\mathrm{d} \lambda / \mathrm{d} n_{\mathrm{s}}$ and $\mathrm{d} \lambda / \mathrm{d} n_{\mathrm{h}}$ and support the fact that the refractive index changes at both interfaces of the permeable thin gold film.

In the second experiment, we tested the structure as a substrate for SERS detection. Accordingly, we probed the upper and bottom interfaces by a laser beam focused from the superstrate or substrate side by a lens with a numerical aperture of 0.5 . The laser beam had a wavelength of $\lambda=785 \mathrm{~nm}$, which is close to the resonance observed at $\lambda_{\mathrm{C}}^{\mathrm{p}}$ associated with the confinement of the incident field in the gap between the NHA and NP (see Fig. 4b). In this experiment, we directly used a structure that was stripped and compared the acquired Raman (Stokes shifted) spectra with that acquired for the same structure in which the upper gold surface and the pore mouth were modified with a Raman-active 4-MBA monolayer (see schematic in Fig. 1b). The results presented in Fig. 7b show a series of Raman peaks in the spectral range of $800-1800 \mathrm{~cm}^{-1}$ for the pristine stripped structure that was probed from the bottom substrate through the pNIPAAm-based cushion. These peaks can be ascribed to the benzophenone molecules at the inner gold interface since their spectral positions are close to that reported in previous works. ${ }^{48}$ After modifying the pore mouth and the upper gold interface with 4-MBA, two additional peaks appeared at $1071 \mathrm{~cm}^{-1}$ (aromatic ring breathing, symmetric $\mathrm{C}-\mathrm{H}$ in-plane bending, and $\mathrm{C}-\mathrm{S}$ stretching) and $1581 \mathrm{~cm}^{-1}$ (aromatic ring $\mathrm{C}-\mathrm{C}$ stretching, asymmetric $\mathrm{C}-\mathrm{H}$ inplane bending). These spectral positions are close to that observed previously for this molecule. ${ }^{48,49}$

Finally, a comparison of the Raman peak intensity was carried out for probing from the top (through the superstrate) and from the bottom (through the pNIPAAm cushion substrate). As can be seen in Fig. 7c, the Raman peaks were observed only for the probing from the bottom, where the mode at $\lambda_{\mathrm{C}}^{\mathrm{p}}$ could be efficiently excited with the laser beam at the wavelength of $785 \mathrm{~nm}$. In addition, the spectral tuning of 
this mode by collapsing and swelling the structure led to variations in the Raman peak intensity. An increase in the Raman peak intensity by about $45 \%$ occurred by collapsing the structure (by drying) with respect to the geometry when the hydrogel cushion was swollen in water. This can be attributed to the potentially stronger field intensity enhancement in the gap between the nanopores in the NHA and the bottom metallic nanoparticles as well as the shift in the resonance at $\lambda_{\mathrm{C}}^{\mathrm{p}}$ to its optimized spectral position with respect to the excitation wavelength and Raman scattered peaks, which was observed to provide most efficient SERS. ${ }^{50-55}$

\section{Conclusions}

We developed a new approach for the preparation of a hybrid plasmonic nanostructure that can be actuated and consists of arrays of nanoholes in a thin gold film, which is connected to arrays of gold nanoparticles by a responsive hydrogel cushion attached to a solid substrate. We explored the spectrum of the plasmonic modes supported by the structure and identified their origin due to the resonant excitation of three localized surface plasmons (confined in the nanopores and at the surface of the nanoparticles) and diffractive coupling to propagating surface plasmons (traveling along the top and bottom interfaces of the thin gold film). By swelling and collapsing the hydrogel cushion, the characteristics of these modes could be changed on-demand and the field confinement as well as resonant wavelength (up to $50 \mathrm{~nm}$ shifts) could be actively actuated. Among the modes, the near-field coupling between the nanoparticles and nanoholes was observed, and the simulations predicted that it leads to strong confinement of the electromagnetic field in the respective gap in the near-infrared part of the spectrum. This is particularly attractive for biosensing applications, as demonstrated by SPR observation of the attachment of $160 \mathrm{kDa}$ IgG molecules inside the structure and SERS measurement of low molecular weight Raman active 4-MBA molecules immobilized in the pore mouth. In addition, this structure offers a unique opportunity to open and close the pores by swelling and collapsing the hydrogel cushion. Accordingly, the pores can be switched between the dead-end geometry and open state when water molecules are actively driven through the pores, dragging dissolved biomolecules across the plasmonic hotspot by diffusion.

\section{Experimental}

\section{Materials}

OrmoStamp® resin was purchased from Micro Resist Technology GmbH (Germany). Ostemer 322 Crystal Clear was purchased from Mercene Labs AB (Sweden). Polydimethylsiloxane Sylgard 184 (PDMS) was obtained from Dow Corning (USA). Trichloro (1H,1H,2H,2H-perfluorooctyl)silane (perfluoro-silane), dimethyl sulfoxide (DMSO) and 1-ethyl-3-(3-dimethylaminopropyl)carbodiimide (EDC) were obtained from Sigma Aldrich (Germany).
(11-Mercaptoundecyl)triethylene glycol (PEG-thiol, SPT-0011) was purchased from SensoPath Technologies Inc. (USA). The pNIPAAm-based terpolymer composed of $N$-isopropylacrylamide, methacrylic acid, and 4-methacryloyloxybenzophenone (in a ratio of $94: 5: 1$ ), benzophenone-disulfide and 4-sulfotetrafluorophenol (TFPS) were synthesized in our laboratory, as previously reported. ${ }^{56,57}$ IgG from mouse serum (mIgG, I 5381) and Tween 20 (P9416) were purchased from Sigma Aldrich (Austria), and phosphate-buffered saline (PBS) and sodium acetate were obtained from VWR Chemicals (Austria). Goat anti-mouse IgG (a-mIgG, A11375) was acquired from Life Technologies, (Eugene OR, US).

\section{UV-nanoimprint lithography}

A template structure bearing arrays of nanopillars was fabricated from a silicon master that carried $1 \mathrm{~cm}^{2}$ rectangular arrays of nanoholes with a diameter of $D=90 \mathrm{~nm}$, depth $260 \mathrm{~nm}$, and period $\Lambda=460 \mathrm{~nm}$, fabricated by Temicon $\mathrm{GmbH}$ (Germany). $200 \mu \mathrm{L}$ of OrmoPrime was spun on a clean BK7 glass substrate at $4000 \mathrm{rpm}$ for $60 \mathrm{~s}$ and hard-baked at $150{ }^{\circ} \mathrm{C}$ for $5 \mathrm{~min}$. The BK7 substrate coated with OrmoPrime was contacted with the silicon master using a drop of OrmoStamp and kept still for 10 min to spread over the structure and fill the pores. The OrmoStamp was cured using UV light at $\lambda=365 \mathrm{~nm}$ with the irradiation dose of $1 \mathrm{~J} \mathrm{~cm}^{-2}$ (UV lamp Bio-Link 365, Vilber Lourmat). Then, the silicon master was carefully detached, leaving the BK7 substrate with an imprinted pattern of nanopillars in the OrmoStamp resin. The fabricated arrays of nanopillars were treated with UV-ozone for 5 min to remove the excess OrmoStamp and activate the surface for silanization. An anti-adhesive layer was deposited on the OrmoStamp structure under an argon atmosphere using $13 \mu \mathrm{L}$ of trichloro( $1 H, 1 H, 2 H, 2 H$-perfluorooctyl)silane in a desiccator (volume $5.8 \mathrm{~L}$ ) heated to $T=250{ }^{\circ} \mathrm{C}$ for $20 \mathrm{~min}$. A $50 \mathrm{~nm}$ thin layer of gold was deposited on the arrays of nanopillars, serving as a template, by vacuum thermal evaporation (HHV AUTO 306 from HHV Ltd) at a deposition rate of $2 \AA \mathrm{s}^{-1}$ in a vacuum greater than $10^{-6}$ mbar. Each sample consisted of a nanostructured region and a flat region for reference in the optical measurements.

\section{Deposition of the responsive polymer}

The OrmoStamp arrays of nanopillars coated with $50 \mathrm{~nm}$ of gold were incubated overnight in a $1 \mathrm{mM}$ solution of benzophenone-disulfide in DMSO to form a self-assembled monolayer serving as a linker. Then, this structure was coated with a uniform layer of pNIPAAm-based terpolymer by spin-coating $3 \mathrm{wt} \%$ ethanolic solution of the polymer at a spin rate of 2000 rpm for $1 \mathrm{~min}$. The layer of the pNIPAAm-based terpolymer was dried overnight under vacuum at $50{ }^{\circ} \mathrm{C}$ yielding, a thickness of $230 \mathrm{~nm}$. The resulting polymer film was crosslinked via the benzophenone moieties by UV light at $\lambda=365 \mathrm{~nm}$ with an irradiation dose of $10 \mathrm{~J} \mathrm{~cm}^{-2}$.

\section{Template stripping}

A drop of Ostemer epoxy was spread on clean BK7 glass by contacting it with a flat piece of PDMS and irradiating it with UV- 
light at $\lambda=365 \mathrm{~nm}\left(2 \mathrm{~J} \mathrm{~cm}^{-2}\right)$. The PDMS block was peeled-off leaving a glass substrate with a flat layer of pre-cured Ostemer epoxy on its top. Then, the Ostemer surface was pressed against the template coated with the crosslinked pNIPAAmbased film and incubated overnight at $50{ }^{\circ} \mathrm{C}$ to allow its attachment to the pNIPAAm-based surface via its epoxy groups. Due to the pre-curing step, the Ostemer did not penetrate the pNIPAAm polymer network layer. Finally, the BK7 substrate with a layer of Ostemer was used to strip off the pNIPAAmbased film with the layer of gold from the template modified with the thin anti-adhesive layer.

\section{Morphological characterization}

Atomic force microscopy (AFM) measurements of the patterned structures in air were performed in tapping mode using PPP-NCHR-50 tips (Nanosensors, Switzerland) and a PicoPlus instrument (Molecular Imaging, Agilent Technologies, USA). In addition, a scanning electron microscope (Zeiss Supra 40 VP (Carl Zeiss Microscopy GmbH, Germany) was used for imaging of the longitudinal and cross-section interfaces of the nanostructures at an electron high tension of EHT $=5 \mathrm{kV}$. The height, diameter and lateral spacing of the nanoscale features were determined using the Gwyddion free software (version 2.47 from gwyddion.net).

\section{Optical configuration for angular-wavelength transmission measurement}

Transmission optical spectra were acquired using a polychromatic light beam emitted from a halogen lamp (LSH102 LOT-Oriel, Germany), which was coupled to a multimode optical fiber and collimated with a lens. It was made incident at the structure and the transmitted beam was collected by a lens to another multimode optical fiber and delivered to a spectrometer (HR4000, Ocean Optics, USA). The obtained transmission spectra were normalized with that obtained on a reference flat $50 \mathrm{~nm}$ thick gold film. A flow-cell with a Peltier element ${ }^{58}$ connected to a controller from Wavelength Electronics Inc. (USA) was clamped against the investigated structure to control the temperature of the liquid flowed over its surface. Deionized water was flowed by employing a peristaltic pump from Ismatec (Switzerland). The investigated structure with a flow cell was mounted on a rotation stage driven by a stepper motor from Huber GmbH (Germany) to control the angle of incident light, $\theta$. Transmission spectra were recorded using the in-house developed Labview software and processed using a dedicated Python script.

\section{Tracking of resonant wavelengths}

Polychromatic light emitted from a supercontinuum laser source (WhiteLaser Micro, Fianium, UK) was collimated and the beam was expanded and spectrally filtered by a long-pass filter. The beam was made incident at a normal angle on the structure mounted in the temperature-stabilized flow cell. The transmitted beam was collected by a GRIN lens to a multimode fiber and delivered to the input of a spectrometer (S2000, Ocean Optics, USA). The acquired transmission spectra were normalized to that measured for a reference flat gold film (thickness of $50 \mathrm{~nm}$ ) and analyzed using the SPR UP software developed at the Institute of Photonics and Electronics, Czech Academy of Sciences.

\section{Finite-difference time-domain simulations}

FDTD simulations were performed using the Lumerical FDTD Solutions software. The geometry of the nanoparticle arrays was described using Cartesian coordinates with the $x$ - and $y$-axis in the plane and the $z$-axis perpendicular to the plane of the arrays. Infinite arrays were considered in the simulations by choosing periodic boundary conditions (symmetric or antisymmetric) along the $x$ - and $y$-axis and using perfectly matched layers (PML) above and below the structure. For the field profile simulations, the simulation mesh was set to $2 \mathrm{~nm}$ over the volume of the unit cell. A transmission monitor was placed $0.4 \mu \mathrm{m}$ below the nanoparticle arrays and a $2 \mathrm{D}$ monitor in the $x z$-plane was employed for simulating the near field distribution of the electric field intensity. The structure was illuminated by a normally incident plane wave with its polarization set along the $x$-direction. The optical constants for $\mathrm{Au}$ were taken from the CRC Optical Data Tables (450-950 nm). To consider the deviations in the experimental geometry from the (idealized) simulated geometry, the diameter $D$ was varied in the range of 100-120 $\mathrm{nm}$ and the respective optical response was averaged.

\section{Immunoassay experiment}

The substrate carrying the NHA + NP structure was clamped against a transparent flow-cell and loaded in an optical system for tracking of the SPR dips or peaks in the transmission spectrum. A polychromatic optical beam was made incident at a normal angle of incidence of $\theta=0^{\circ}$ at the structure, and by analysis of the transmitted light spectrum, the variations in the resonant wavelengths were monitored with time. These variations were determined by fitting the acquired spectrum with a polynomial function, as reported previously, ${ }^{47}$ and which allowed the spectral shifts of the dip or peak features to be measured with the accuracy of 0.1-0.01 nm, depending on the coupling strength and noise in the transmission spectrum. Firstly, the baseline in the resonant wavelength kinetics was established upon a flow of PBS for $20 \mathrm{~min}$. Then, a mixture of EDC/TFPS dissolved in water at concentrations 75 and $21 \mathrm{mg}$ $\mathrm{mL}^{-1}$, respectively, was flowed over the structure for $10 \mathrm{~min}$ to activate the carboxylic moieties of the pNIPAAm hydrogel. The surface was quickly rinsed with $\mathrm{pH} 5$ acetate buffer and reacted with a solution of $50 \mu \mathrm{g} \mathrm{mL} \mathrm{mL}^{-1}$ mouse IgG in the same buffer for $60 \mathrm{~min}$ to covalently attach the mIgG molecules to the polymer chains. Finally, the structure was rinsed with PBS, followed by the flow of PBS spiked with $2 \%, 4 \%$ and $8 \%$ sucrose $\left(\Delta n=2.8 \times 10^{-3}, 5.6 \times 10^{-3}\right.$ and $11.2 \times 10^{-3} \mathrm{RIU}$, respectively).

\section{SERS experiments}

The NHA + NP structure was incubated overnight in $1 \mathrm{mM}$ ethanolic solution of 4-mercaptobenzoic acid to form a self- 
assembled monolayer of SERS-active molecules. Prior to the experiment, the structure was rinsed with ethanol and dried. The SERS experiments were performed using an Xplora Raman microspectrometer (Horiba Scientific, France) with a $\times 50$ long working distance objective (numerical aperture of 0.5 ). The laser beam at $\lambda=785 \mathrm{~nm}$ was focused at the investigated NHA + NP structure. The spectrum was accumulated for $20 \mathrm{~s}$.

\section{Conflicts of interest}

There are no conflicts to declare.

\section{Acknowledgements}

DK acknowledges funding from the European Union's Horizon 2020 research and innovation programme under grant agreement no. 642787, Marie Sklodowska-Curie Innovative Training Network BIOGEL. SF, JS, JD, JH and UJ were supported by European Union's Horizon 2020 research and innovation programme under grant agreement no. 633937, project ULTRAPLACAD. PV, ML, and MDLC are grateful for the financing from the project jointly funded by Agence Nationale de la Recherche (ANR) and Austrian Science Fund (FWF) under the grant agreements ANR-15-CE29-0026 and I 2647, respectively. SF and JD received support by the Austrian Research Promotion Agency (FFG) with grant agreement no. 861578 (ERANET project PLABAN). JS and JH acknowledge financial support from the Czech Science Foundation, grant agreement \#19-02739S. The Raman analysis was performed at the Vibrational Spectroscopy platform of the IMMM, Universite du le Mans.

\section{Notes and references}

1 T. W. Ebbesen, H. J. Lezec, H. F. Ghaemi, T. Thio and P. A. Wolff, Nature, 1998, 391, 667-669.

2 M. Najiminaini, F. Vasefi, B. Kaminska and J. J. L. Carson, Sci. Rep., 2013, 3, 1-7.

3 M. S. Ahn, T. Chung and K. H. Jeong, Nanoscale, 2018, 10, 6313-6317.

4 A. G. Brolo, S. C. Kwok, M. G. Moffitt, R. Gordon, J. Riordon and K. L. Kavanagh, J. Am. Chem. Soc., 2005, 127, 14936-14941.

5 Q. Zhang, L. Wu, T. I. Wong, J. Zhang, X. Liu, X. Zhou, P. Bai, B. Liedberg and Y. Wang, Int. J. Nanomed., 2017, 12, 2307-2314.

6 A. G. Brolo, E. Arctander, R. Gordon, B. Leathem and K. L. Kavanagh, Nano Lett., 2004, 4, 2015-2018.

7 P. Zheng, S. K. Cushing, S. Suri and N. Wu, Phys. Chem. Chem. Phys., 2015, 17, 21211-21219.

8 S. Kumar, S. Cherukulappurath, T. W. Johnson and S. Oh, Chem. Mater., 2014, 26, 6523-6530.

9 T. Wu and Y. W. Lin, Appl. Surf. Sci., 2018, 435, 1143-1149.
10 S. P. Sahu, A. Mahigir, B. Chidester, G. Veronis and M. R. Gartia, Nano Lett., 2019, 19, 6192-6202.

11 M. E. Stewart, N. H. Mack, V. Malyarchuk, J. A. N. T. Soares, T.-W. Lee, S. K. Gray, R. G. Nuzzo and J. A. Rogers, Proc. Natl. Acad. Sci. U. S. A., 2006, 103, 1714317148.

12 A. A. Yanik, A. E. Cetin, M. Huang, A. Artar, S. H. Mousavi and A. Khanikaev, Proc. Natl. Acad. Sci. U. S. A., 2011, 108, 11784-11789.

13 K. Nakamoto, R. Kurita, O. Niwa, T. Fujii and M. Nishida, Nanoscale, 2011, 3, 5067-5075.

14 X. Li, M. Soler, C. I. Özdemir, A. Belushkin, F. Yesilköy and H. Altug, Lab Chip, 2017, 17, 2208-2217.

15 H. Im, H. Shao, Y. I. Park, V. M. Peterson, C. M. Castro, R. Weissleder and H. Lee, Nat. Biotechnol., 2014, 32, 490495.

16 A. A. Yanik, M. Huang, O. Kamohara, A. Artar, T. W. Geisbert, J. H. Connor and H. Altug, Nano Lett., 2010, 10, 4962-4969.

17 J. A. Jackman, E. Linardy, D. Yoo, J. Seo, W. B. Ng, D. J. Klemme, N. J. Wittenberg, S. H. Oh and N. J. Cho, Small, 2016, 12, 1159-1166.

18 J. Gomez-Cruz, S. Nair, A. Manjarrez-Hernandez, S. Gavilanes-Parra, G. Ascanio and C. Escobedo, Biosens. Bioelectron., 2018, 106, 105-110.

19 L. Tu, X. Li, S. Bian, Y. Yu, J. Li, L. Huang, P. Liu, Q. Wu and W. Wang, Sci. Rep., 2017, 7, 11020.

20 J. T. Lim, Y. S. Yoon, W. Y. Lee, J. T. Jeong, G. S. Kim, T. G. Kim and S. K. Lee, Nanoscale, 2017, 9, 17224-17232.

21 S. Kumar, G. G. Wolken, N. J. Wittenberg, E. A. Arriaga and S. H. Oh, Anal. Chem., 2015, 87, 1973-11977.

22 M. P. Jonsson, P. Jönsson, A. B. Dahlin and F. Höök, Nano Lett., 2007, 7, 3462-3468.

23 L. Plucinski, M. Ranjan, W. R. Arnold, A. Ameen, T. Chang, A. Hsiao, G. Logan and A. Das, Biosens. Bioelectron., 2016, 75, 337-346.

24 W.-C. Liu and T. D. Ping, Phys. Rev. B: Condens. Matter Mater. Phys., 2002, 65, 155423.

25 F. J. Garcia-Vidal, L. Martin-Moreno, T. W. Ebbesen and L. Kuipers, Rev. Mod. Phys., 2010, 82, 729-787.

26 M. Horak, V. Krapek, M. Hrton, A. Konecna, F. Ligmajer, M. Stoeger-Pollach, T. Samoril, A. Patak, Z. Edes, O. Metelka, J. Babocky and T. Sikola, Sci. Rep., 2019, 9, 4004.

27 L. Wang, B. Xu, W. Bai, J. Zhang, L. Cai, H. Hu and G. Song, Plasmonics, 2012, 7, 659-663.

28 C. Stelling and M. Retsch, Adv. Mater. Interfaces, 2018, 5, 17.

29 R. Mohammadi, M. Ochs, A. Andrieu-Brunsen and N. Vogel, J. Phys. Chem. C, 2020, 124, 2609-2618.

30 M. Hentschel, T. Weiss, S. Bagheri and H. Giessen, Nano Lett., 2013, 13, 4428-4433.

31 V. G. Kravets, A. V. Kabashin, W. L. Barnes and A. N. Grigorenko, Chem. Rev., 2018, 118, 5912-5951.

32 J. F. Masson, M. P. Murray-Méthot and L. S. Live, Analyst, 2010, 135, 1483-1489. 
33 C. Escobedo, Lab Chip, 2013, 13, 2445-2463.

34 H. Im, S. H. Lee, N. J. Wittenberg, T. W. Johnson, N. C. Lindquist, P. Nagpal, D. J. Norris and S. H. Oh, ACS Nano, 2011, 5, 6244-6253.

35 S. H. Lee, K. C. Bantz, N. C. Lindquist, S.-H. Oh and C. L. Haynes, Langmuir, 2009, 25, 13685-13693.

36 B. Ai, Y. Yu, H. Möhwald, G. Zhang and B. Yang, Adv. Colloid Interface Sci., 2014, 206, 5-16.

37 J. Junesch, T. Sannomiya and A. B. Dahlin, ACS Nano, 2012, 6, 10405-10415.

38 H. Im, J. N. Sutherland, J. A. Maynard and S.-H. Oh, Anal. Chem., 2012, 84, 1941-1947.

39 A. Barik, L. M. Otto, D. Yoo, J. Jose, T. W. Johnson and S. H. Oh, Nano Lett., 2014, 14, 2006-2012.

40 J. Dostálek and W. Knoll, Plasmonics, 2012, vol. 2.

41 Y. Zhao, G. Gaur, S. T. Retterer, P. E. Laibinis and S. M. Weiss, Anal. Chem., 2016, 88, 10940-10948.

42 C. Escobedo, A. G. Brolo, R. Gordon and D. Sinton, Anal. Chem., 2010, 82, 10015-10020.

43 N. Sharma, C. Petri, U. Jonas and J. Dostalek, Opt. Express, 2016, 24, 2457.

44 N. Gisbert Quilis, M. van Dongen, P. Venugopalan, D. Kotlarek, C. Petri, A. Moreno Cencerrado, S. Stanescu, J. L. Toca Herrera, U. Jonas, M. Möller, A. Mourran and J. Dostalek, Adv. Opt. Mater., 2019, 7, 1-11.

45 N. Sharma, H. Keshmiri, X. Zhou, T. I. Wong, C. Petri, U. Jonas, B. Liedberg and J. Dostalek, J. Phys. Chem. C, 2016, 120, 561-568.

46 A. Aulasevich, R. F. Roskamp, U. Jonas, B. Menges, J. Dostálek and W. Knoll, Macromol. Rapid Commun., 2009, 30, 872-877.
47 A. T. Reiner, N. G. Ferrer, P. Venugopalan, R. C. Lai, S. K. Lim and J. Dostálek, Analyst, 2017, 142, 3913-3921.

48 Y. Fleger, Y. Mastai, M. Rosenbluh and D. H. Dressler, Surf. Sci., 2009, 603, 788-793.

49 N. G. Quilis, M. Lequeux, P. Venugopalan, I. Khan, W. Knoll, S. Boujday, M. L. de la Chapelle and J. Dostalek, Nanoscale, 2018, 10, 10268-10276.

50 S. J. Lee, Z. Guan, H. Xu and M. Moskovits, J. Phys. Chem. C, 2007, 111, 17985-17988.

51 M. Chirumamilla, A. Gopalakrishnan, A. Toma, R. P Zaccaria and R. Krahne, Nanotechnology, 2014, 25, 235303.

52 N. Guillot and M. L. de la Chapelle, J. Quant. Spectrosc. Radiat. Transfer, 2012, 113, 2321-2333.

53 N. Guillot, B. Fremaux, H. Shen, O. Péron, E. Rinnert, T. Toury and M. Lamy De La Chapelle, Appl. Phys. Lett., 2010, 97, 023113.

54 S. Kessentini, D. Barchiesi, C. D’Andrea, A. Toma, N. Guillot, E. Di Fabrizio, B. Fazio, O. M. Maragó, P. G. Gucciardi and M. Lamy De La Chapelle, J. Phys. Chem. C, 2014, 118, 3209-3219.

55 F. J. Colas, M. Cottat, R. Gillibert, N. Guillot, N. Djaker, N. Lidgi-Guigui, T. Toury, D. Barchiesi, A. Toma, E. Di Fabrizio, P. G. Gucciardi and M. L. De La Chapelle, J. Phys. Chem. C, 2016, 120, 13675-13683.

56 P. W. Beines, I. Klosterkamp, B. Menges, U. Jonas and W. Knoll, Langmuir, 2007, 23, 2231-2238.

57 K. Sergelen, C. Petri, U. Jonas and J. Dostalek, Biointerphases, 2017, 12, 051002.

58 M. Toma, U. Jonas, A. Mateescu, W. Knoll and J. Dostalek, J. Phys. Chem. C, 2013, 117, 11705-11712. 